\section{Increased usage of botulinum toxin and hyaluronic acid fillers in young adults}

Dear Editor,

Over the past decades, dermal fillers and botulinum toxin have become broadly available for the improvement of undesirable skin wrinkles and sagging. ${ }^{1}$ Previous studies have mentioned a range of factors resulting in patient motivations for non-surgical aesthetic treatments. Among these were social awareness, acceptance of cosmetic treatments, a growing sociocultural emphasis on beauty and self-image or self-esteem..$^{2-4}$ Furthermore, both women and men are increasingly affected by beauty ideals, presented by the mass media, resulting in more dissatisfaction about their self-appearance. ${ }^{5}$ In pursuit of physical perfections, the risk of adverse cutaneous reactions may increase due to addiction to cosmetic injections. ${ }^{6}$ A higher engagement of social media usage has been linked to self-image dissatisfaction. ${ }^{7}$ Especially since the majority of social media users are young adults, the question arises whether this group is a growing user of aesthetic treatments such as botox and fillers. ${ }^{8}$ According to a 2018 survey by the American Academy of Facial Plastic and Reconstructive Surgery, $72 \%$ of facial plastic surgeons saw an increase in cosmetic surgery or injectables in patients under the age of 30 . This would be an increase of $24 \%$, compared to 2013 . $^{\circ}$ Yet to our knowledge, no study has used a clinical database to assess this trend.

The aim of this study is to quantify the trend of incidence of young adults choosing for non-surgical cosmetic treatments in a multi-centre setting. The Electronic Health Reports (EHR) of three medical centres in the Netherlands were examined in this multi-centre retrospective observational study: Velthuis Kliniek, Nationaal Huidcentrum and Kliniek Veldhoven. These medical centres give a representative view on a nationwide trend since they contain both high-end locations and accessible ones. Added together, the acquired data originates from ten locations spread across the Netherlands. All-time anonymously data of patients consulting an aesthetic physician for botulinum toxin and hyaluronic acid fillers treatment were considered for inclusion. Only the first consultation of each individual patient was included in this study, excluding check-up appointments and new visits by already registered patients. Young adults are defined as the age group 18-25 years old in this study, based on our clinical categorization of this group.

A total of 12628 patients were included, spread over the years 2008-2019. Table 1 illustrates the numbers included per year. A number of 8453 patients were excluded due to missing or inaccurate data with respect to age or date of consultation. These missing data were not at random and were more common in the far past due to poorer documentation. No other specific bias was found as a cause. Concerning age, 584 patients (4.6\%) fell under the age category of 18-25 years, while 12044 (95.4\%) patients were 26 years or older. Over the eleven years, the share of young adults was significantly correlated with the year of initial consultation $\chi(11)=62.282, P<0.01$. Figure 1 shows the trend in share of young adults, compared to the total annual percentage of patients. In most years, the share of young adults has increased in the use of botulinum toxin and hyaluronic acid

Table 1 Trend in years

\begin{tabular}{|c|c|c|c|c|c|c|c|c|c|}
\hline & & \multicolumn{4}{|c|}{ Age group } & \multicolumn{4}{|c|}{ Gender } \\
\hline & & \multicolumn{2}{|c|}{ Age $>25$} & \multicolumn{2}{|c|}{ Age 18-25 } & \multicolumn{2}{|c|}{ Female } & \multicolumn{2}{|c|}{ Male } \\
\hline & & $n$ & $\%$ & $n$ & $\%$ & $n$ & $\%$ & $n$ & $\%$ \\
\hline \multirow[t]{12}{*}{ Year of initial consultation } & 2008 & 158 & 96.9 & 5 & 3.1 & 60 & 88.2 & 8 & 11.8 \\
\hline & 2009 & 326 & 97.0 & 10 & 3.0 & 129 & 86.0 & 21 & 14.0 \\
\hline & 2010 & 292 & 97.7 & 7 & 2.3 & 110 & 88.7 & 14 & 11.3 \\
\hline & 2011 & 917 & 97.9 & 20 & 2.1 & 530 & 94.3 & 32 & 5.7 \\
\hline & 2012 & 583 & 95.9 & 25 & 4.1 & 273 & 85.8 & 45 & 14.2 \\
\hline & 2013 & 527 & 97.1 & 16 & 2.9 & 230 & 92.4 & 19 & 7.6 \\
\hline & 2014 & 880 & 95.8 & 39 & 4.2 & 157 & 88.7 & 20 & 11.3 \\
\hline & 2015 & 1007 & 95.4 & 49 & 4.6 & 186 & 89.0 & 23 & 11.0 \\
\hline & 2016 & 761 & 96.9 & 24 & 3.1 & 206 & 88.0 & 28 & 12.0 \\
\hline & 2017 & 940 & 92.0 & 82 & 8.0 & 222 & 84.7 & 40 & 15.3 \\
\hline & 2018 & 2087 & 94.2 & 129 & 5.8 & 284 & 86.9 & 43 & 13.1 \\
\hline & 2019 & 3566 & 95.2 & 178 & 4.8 & 275 & 84.6 & 50 & 15.4 \\
\hline
\end{tabular}




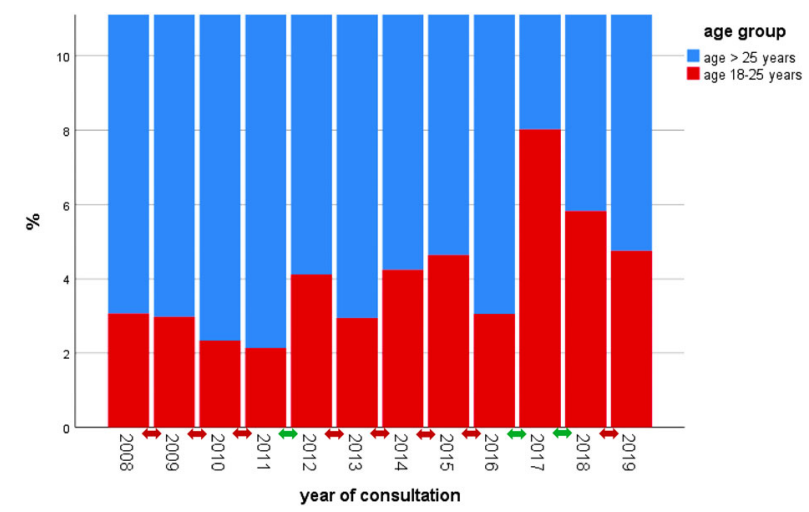

Figure 1 This figure illustrates the percentage of young adults per year. The arrows in between the years represent the statistical significance of the chi-square test between the two adjacent years. A green arrow highlights a statistical significance of $P<0.05$. The exact numbers are as follows: (i) 2008-2009: $\chi(1)=0.003$, $P=0.955$. (ii) 2009-2010: $\chi(1)=0.245, P=0.621$. (iii) 2010-2011: $\chi(1)=0.045, P=0.831$. (iv) 2011-2012: $\chi(1)=5.099, P=0.024$. (v) 2012-2013: $\chi(1)=1.134, P=0.287$. (vi) 2013-2014: $\chi$ (1) $=1.586, P=0.208$. (vii) 2014-2015: $\chi(1)=0.181, P=0.670$. (viii) 2015-2016: $\chi(1)=2.962, P=0.085$. (ix) 2016-2017: $\chi$ (1) = 19.829, $P<0.000$. (x) 2017-2018: $\chi(1)=5.568, P=0.018$. (xi) 2018-2019: $\chi(1)=3.244, P=0.072$.

fillers. It is remarkable that the year 2017 forms a significant peak in the share of young adults. Added up, 343 men visited an aesthetic physician compared to 2662 women, forming a $11.4 \%$ minority. A statistically significant correlation was found between the year of the appointment and gender, $\chi(11)=35.065$, $P<0.01$. As Table 1 illustrates, the share of men tends to fluctuate annually while increasing in the more recent years.

The interpretation of these data remains a point of discussion, since these data are an illustration of the concerning years and the usage of these treatments may be yeardependent. However, the current data do support previous questionnaires among plastic surgeons pointing out the increasing use of botulinum toxin and hyaluronic acid fillers usage in young adults. ${ }^{10}$ In conclusion, for the first time the use of these treatments by young adults and men has been expressed in multi-centre clinical data.

\section{Funding sources}

The authors declare no financial interest in the subject relevant to the manuscript. This study has no involvement of study sponsors.

\section{Ethical approval}

Research concerning anonymously non-traceable data does not require approval by an ethics committee according to Dutch law (WMO).

\section{Zarringam, ${ }^{1}$ (iD T. Decates, ${ }^{1, *}$ (iD H.P. Slijper, ${ }^{2}$ P. Velthuis ${ }^{1}$ \\ ${ }^{1}$ Department of Dermatology, Erasmus Medical Center, Rotterdam, The Netherlands, ${ }^{2}$ Velthuis Kliniek, Eindhoven, The Netherlands \\ ${ }^{*}$ Correspondence: T. Decates. E-mail: info@drdecates.com}

\section{References}

1 Kelly PE. Injectable success: from fillers to Botox. Facial Plast Surg 2007; 23: 7-18.

2 Von Soest T, Kvalem IL, Skolleborg KC, Roald HE. Psychosocial factors predicting the motivation to undergo cosmetic surgery. Plast Reconstr Surg2006; 117: 51-62.

3 Haas CF, Champion A, Secor D. Motivating factors for seeking cosmetic surgery: a synthesis of the literature. Plast Surg Nurs 2008; 28: 177-182.

4 Milothridis P, Pavlidis L, Haidich AB, Panagopoulou EA. Systematic review of the factors predicting the interest in cosmetic plastic surgery. Indian J Plast Surg 2016; 49: 397-402.

5 Barlett CP, Vowels CL, Saucier DA. Meta-analyses of the effects of media images on men's body-image concerns. J Soc Clin Psychol 2008; 27: 279-310.

6 Rongioletti F, Atzori L, Ferreli Cet al. Granulomatous reactions after injections of multiple aesthetic micro-implants in temporal combinations: a complication of filler addiction. J Eur Acad Dermatol Venereol 2015; 29: 1188-1192.

7 Mclean SA, Paxton SJ, Wertheim EH, Masters J. Photoshopping the selfie: Self photo editing and photo investment are associated with body dissatisfaction in adolescent girls. Int J Eat Disord 2015; 48: 1132-1140.

8 Thackeray R, Crookston BT, West JH. Correlates of health-related social media use among adults. J Med Internet Res 2013; 15: e21.

9 American Academy of Facial Plastic and Reconstructive Surgery. 2018 Annual Survey Statistics. [Online]. URL www.aafprs.orgwww.legac y.aafprs.org/media/stats_polls/m_stats.html. Published January 29, 2018. (last accessed: 6 March 2018).

10 American Society for Aesthetic Plastic Surgery. Cosmetic (Aesthetic) Surgery National Data Bank STATISTICS. [Online]. URL https://www.surge ry.org/sites/default/files/ASAPS-Stats2018_0.pdf (last accessed: 10 November 2019).

DOI: $10.1111 / \mathrm{jdv} .16481$ 